\title{
la réparation du béton par injection examen de deux cas particuliers
}

\author{
par \\ A. Mayer \\ Ingénieur Général des Mines (e.r.) \\ Président d'Honneur de Comité Français de Mécanique des Roches \\ et \\ C. Caron \\ Ingénieur Docteur \\ Président du Groupe d'Études et d'Innovations pour la Construction
}

L'augmentation des contraintes appliquées au béton dans les constructions nouvelles, ainsi que le développement du béton précontraint ont causé de nombreux incidents dus à des contraintes locales excessives et du fait de l'apparition de fissures réduisaient la résistance et dans certains cas l'étanchéité des ouvrages. Ces fissures peuvent également être causées par des mouvements du sol dus, soit à des tassements, soit à des séismes. II est immédiatement apparu que la réparation des fissures et la reconstitution de l'homogénéité du béton pouvaient être obtenus par injections. Cette technique paraissait d'autant plus indiquée que, dans le cas notamment des ouvrages de retenues hydrauliques, le terrain sous-jacent était fréquemment et avec succès consolidé par injection et qu'à priori on pouvait assimiler un rocher fissuré à un béton fissuré.

L'expérience qui date de plus d'un siècle a démontré l'efficacité des injections de ciment pour la consolidation du rocher fissuré ou fracturé des massifs karstiques. La théorie et la pratique se conjuguent pour montrer la nécessité d'une montée en pression dans ce type de traitement. II faut en effet ouvrir les fissures et essorer le coulis de ciment, ce qui nécessite une mise en charge suffisante. Or, pour que cette montée en pression soit possible et efficace, il faut une certaine couverture.

Lugeon, dans son ouvrage "Barrages et géologie» signalait déjà en 1933 le peu d'efficacité de l'injection de ciment dans les couches géologiques proches de la surface et Cambefort (Bulletin Technique de la Suisse Romande $\left.n^{\circ} 19,1961\right)$ montrait que les désordres intervenus dans les fondations de certains barrages au Maroc étaient imputables à des pressions d'injections trop faibles. Dans le corps du barrage lui-même, l'injection à haute pression est impossible. II faut donc renoncer au traitement par des coulis de ciment dilué et recourir à des coulis stables injectables a basse pression qui seront en général des résines. L'étude des différentes résines artificielles a en effet permis la mise au point de matériaux obtenus en mélangeant une résine et un réactif lesquels sont aussi fluides que l'eau pendant toute l'injection et qui durcissent progressivement tandis que leurs propriétés mécaniques augmentent jusqu'à devenir parfois superieures à celles du béton.

La reconstitution de la résistance initiale d'un béton fissuré peut être obtenue avec deux types différents de résines, les polyesters et les épicotes. Après prise, leur résistance ainsi que leurs propriétés adhésives sont comparables. Les épicotes se contractent moins après prise, leur pouvoir adhésif est un peu meilleur, mais ils sont plus visquieux. C'est pourquoi on les utilise en général dans des fissures plus larges (1 $\mathrm{mm}$ ou plus) tandis que les polyesters sont employés dans les plus fines.

Théoriquement il n'y a pas de limite à la possibilité d'injection de ces résines fluides; tout en opérant à basse pression il suffit d'allonger le temps de prise pour pouvoir traiter des fissures de plus en plus fines. Au barrage de Tolla, en Corse, il a été possible d'obturer des fissures inférieures à $1 / 10 \mathrm{~mm}$ avec un polyester fluide à temps de prise de 20 heures.

Mais ces hautes performances des résines ne doivent pas faire oublier le ciment chaque fois que cela est possible c'est-à-dire lorsque le béton de l'ouvrage est assez perméable et que l'on cherche plus une préservation qu'une consolidation. Ce serait le cas d'un béton délavé qu'il faut préserver des dissolutions ultérieures. Les coulis à base de ciment utilisés dans ces cas sont des coulis stables qu'il n'est pas nécessaire d'essorer sous pression.

On voit ainsi que le choix entre ces deux méthodes dépend essentiellement de deux conditions : - la nature du béton à régénérer, fissures, zones
délavées...

- le but à atteindre : une haute consolidation ou la préservation.

Si ces cas peuvent être traités par l'emploi des coulis à base de ciment il faut évidemment y songer, car ce type d'injection est beaucoup plus économique que celui des résines.

Afin de préciser ces deux types de réparation, nous prendrons deux exemples:

- le premier concerne un barrage endommagé par un tremblement de terre et dont les fissures ont été injectées avec des résines époxydes et des polyesters.

- le second concerne un barrage de plus de 60 ans d'âge construit en béton de chaux hydraulique. La dissolution partielle de celle-ci avait conduit à des phénomènes inadmissibles qui auraient à la longue compromis la stabilité de l'ouvrage. Le bouchage par injection de ciment stabilisé de tous les vides de l'ouvrage a permis de lui rendre une nouvelle jeunesse. 


\section{Réparation du barrage de Koyna près de Bombay (Inde)}

Le barrage de Koyna a été construit vers 1960 a 200 km au Sud de Bombay, à la limite du plateau du Décan. Ce plateau est constitué par des niveaux du début de l'ère secondaire complètement recouverts par la coulée de basalte qui s'est répandue sur toute la partie centrale de I'Inde. Jusqu'à l'accident de Koyna cette zone avait été considérée comme pratiquement à l'abri des tremblements de terre, tout au moins comparée à différentes régions du Nord de I'Inde.

Des recherches ont été entreprises après l'accident et ont montré qu'il y avait eu dans la région de Bombay 23 tremblements de terre au cours des 400 dernières années, dont un en 1618 qui détruisit la plus grande partie de Bombay. Mais alors qu'on en avait noté 13 entre 1751 et 1764 , il n'en était arrivé aucun depuis, si bien que le projet du barrage avait été fait en tenant compte d'une accélération horizontale de $0,05 \mathrm{~g}$ ce qui correspond à une probabilité très faible. Le barrage est un barrage poids de $80 \mathrm{~m}$ de hauteur construit avec grand soin en utilisant un excellent béton cyclopéen. La face amont est tout à fait plane tandis que la face aval est divisée en deux parties, l'une correspondant au $1 / 3$ supérieur, presque verticale, l'autre sur les $2 / 3$ inférieurs avec une pente de l'ordre de $60^{\circ}$.

Dès que le réservoir fut rempli en 1962 des petites secousses furent ressenties dans la région. Des sismographes furent mis en place à l'intérieur et autour du barrage. Jusqu'en Septembre 1967, 154 secousses ont été ressenties d'une intensité comprise entre 2 et 3 sur l'échelle de Richter. Les épicentres étaient sous le réservoir, à une profondeur comprise entre 3 et $5000 \mathrm{~m}$. L'activité sismique ne cessa d'augmenter jusqu'au 10 Décembre 1967 où se produisit un tremblement de terre entre 6,5 et 7,5 sur l'échelle de Richter. L'épicentre était à $3 \mathrm{~km}$ au Sud du barrage et l'ébranlement affecta une zone de $600 \mathrm{~km}$ de rayon environ. II causa d'importants dommages au village construit à proximité de Koyna et provoqua la rupture des câbles qui reliaient la centrale souterraine à la ville de Bombay plongeant une grande partie de la ville dans l'obscurité. Le barrage lui-même ne subit pas de dégâts très importants et le réservoir put être maintenu en eau, toutefois à un niveau inférieur au maximum. Le long de la ligne correspondant au changement de pente de la face aval il était apparu une fissure horizontale dans les plots du centre du barrage. Dans deux de ceux-ci on constata des résurgences à l'aval pendant la secousse ce qui prouvait que la fissure traversait entièrement ces deux plots. Sur les 31 plots constituant le barrage 14 étaient fissurés. Des forages exécutés à partir de la crête et comportant une injection d'eau montrèrent que les fissures, bien qu'apparaissant dans différents plots sur les deux faces du barrage, ne traversaient l'ouvrage de part en part que dans les deux plots centraux.

A la requête du Gouvernement de l'Inde, l'UNESCO envoya deux missions pour étudier la situation. L'une dirigée par le Professeur O. Kamoto de Tokio devait étudier l'aspect sismique du phéonomène et le risque d'une répétition d'un accident analogue. La seconde, comprenant $M$. Bellier, Chef du Bureau d'Ingénieurs-Conseils Coyne et Bellier et l'un des auteurs de ces lignes, devait émettre un avis sur l'état de l'ouvrage et proposer les mesures à prendre pour rétablir l'homogénéité du barrage de façon à permettre son utilisation et lui donner la possibilité de résister à un tremblement de terre d'une intensité comparable à celui de 1967.

Trois dispositifs furent proposés pour réparer les désordres et pour accroître la stabilité de l'ouvrage de manière à lui permettre de résister à un ébranlement d'intensité $0,8 \mathrm{~g}$ comme celui qui s'était produit, au lieu de $0,05 \mathrm{~g}$ dont on avait tenu compte lors des études. C'étaient :

- le bouchage des fissures par injection de résines,

- l'introduction de câbles traversant l'ouvrage, ancrés dans le rocher de fondation, et précontraints,

- l'élargissement de la base de l'ouvrage en augmentant l'épaisseur de sa partie inférieure amont.
Les deux dernières propositions avaient pour objet d'augmenter la sécurité de l'ouvrage. Mais le plus urgent était de boucher les fissures de façon que, si un nouveau séisme se produisait après la mousson, c'est-à-dire à quelques mois de notre visite sur les lieux, et une fois le réservoir à sa cote maxima, les dégâts ne soient pas pires que ceux de 1967.

II fut alors proposé à I'UNESCO de faire venir immédiatement $M$. Caron, spécialiste en matière d'injections de résines, de manière à boucher la fissure avant la mousson.

Cette proposition fut acceptée. M. Caron vint à Koyna et prit en main la direction du travail. II disposait sur place des filiales locales de deux entreprises européennes spécialisées. Il fit les études de laboratoire nécessaires pour déterminer parmi les résines disponibles sur place, celles qui convenaient le mieux dans le cas particulier. II définit ainsi deux méthodes de traitement.

a) fissures peu profondes

C'est ce type de fissures que l'on avait constatées dans la partie centrale de l'ouvrage. Elles étaient visibles sur une longueur de $100 \mathrm{~m}$ sur la face amont et $150 \mathrm{~m}$ sur la face aval. Elles ont été bouchées par injection d'une résine épicote, grâce à une série de trous courts à environ $1 \mathrm{~m}$ de distance l'un de l'autre. Comme les fissures étaient peu profondes et que la distance entre deux trous successifs était faible, la quantité de matière injectée dans chaque trou fut faible. On put utiliser un matériel léger, un coulis assez visqueux et un temps de prise court. On put trouver des résines époxy produites sur place qui permirent d'obtenir ces résultats.

b) fissures traversant les plots

Comme il a été dit on ne trouva ce type de fissure que sur deux plots. Le temps dont on disposait pour exécuter le travail était très court, si on voulait le terminer avant la mousson. On décida donc d'utiliser un matériel lourd, une résine plus fluide et un temps de prise plus long de manière à réduire le nombre de forages nécessaires et d'exécuter ceux-ci verticalement à partir de la crête de l'ouvrage. Après avoir bouché, comme il a été dit ci-dessus, les fissures au voisinage des parois, on injecta un coulis de polyester dans l'intervalle entre les deux fissures extérieures. Le coulis remplit complètement le vide correspondant à la partie centrale de la fissure et des essais effectués sur des carottes prélevées après prise montrèrent que les deux parties des plots fissurés avaient été recollées de manière à avoir retrouvé une résistance continue convenable.

La mousson survint quelques mois après. Le réservoir fut rempli jusqu'au niveau du déversoir sans que l'on put constater le moindre déplacement de la partie supérieure des plots recollés. Depuis, des examens très complets ont démontré le comportement très satisfaisant de l'ouvrage.

L'exemple du barrage de Koyna est probablement le plus frappant de ceux dont les soussignés ont eu connaissance mais on peut indiquer que la même technique a été appliquée avec succès au cours des dernières années, tant en France qu'à l'étranger, sur des bâtiments, des ponts, des écluses et des barrages où s'étaient produites des fissures qu'il y avait lieu de boucher. La technique des injections à la résine est maintehant bien connue et peut être appliquée sans arrière pensée.

\section{Remise en état du barrage de Dardennes près de Toulon}

L'exemple qui sera développé ci-après est celui de la réparation du barrage de Dardennes près de Toulon, grâce à des injections de coulis de ciment. Le cas était très exceptionnel. Le barrage est en effet situé dans les hauteurs dominant la ville de Toulon et un accident survenant à si courte distance en amont d'une ville aussi importante aurait eu des conséquences dramatiques. C'est pourquoi, il y a une dizaine d'années, en raison des résurgences qui apparaissaient sur la face aval de 
l'ouvrage, la Ville de Toulon décida de faire examiner l'état de l'ouvrage et de rechercher la cause des fuites. L'auteur fut chargé de ces travaux et fit appel à la Société SOLETANCHE pour faire un certain nombre de forages le long de la crête de l'ouvrage pour reconnaître l'état de la maçonnerie.

Le barrage de Dardennes est un barrage-poids en béton de chaux hydraulique construit aux environs de 1910 d'une hauteur maxima de $36 \mathrm{~m}$ et destiné à alimenter la ville de Toulon en eau potable. Dès les premiers forages on constata qu'une partie du liant constitutif de l'ouvrage avait été délavé et qu'il y avait à l'intérieur de l'ouvrage un niveau d'eau correspondant exactement à celui de la retenue. Chose surprenante, lorsque l'on baissait le niveau dans la retenue on ne constatait aucune résurgence sur la face amont, ce qui n'aurait pas manqué de se produire si l'étanchéité du revêtement de cette face n'avait pas été complète. Le faible débit des résurgences de la face aval montrait que celle-ci était également imperméable à part quelques points isolés. L'alimentation de la nappe à l'intérieur de l'ouvrage devait se produire par infiltration 'sous le masque amont qui n'était ancré dans le rocher qu'à faible profondeur et l'étanchéité de l'ouvrage était assurée du fait du masque aval en maçonnerie appareillée. La situation apparaissait extrêmement inquiétante et la Municipalité décida de faire exécuter immédiatement les travaux confortatifs nécessaires. La dimension des vides à l'intérieur de l'ouvrage était suffisante pour que l'on puisse procéder a leur obturation par injections de coulis de ciment à basse pression. On décida de forer un trou tous les trois mètres le long de la crête et de l'injecter à une pression inférieure a $0,5 \mathrm{MPa}$. Lorsque ceci fut fait' des forages de contrôle furent exécutés entre les premiers et équipés en piézomètres. On injecta ainsi plusieurs centaines de tonnes de ciment dans les vides du béton.
Une fois ce travail terminé, les piézomètres montrèrent que le niveau d'eau à l'intérieur du barrage avait complètement disparu. II faut noter que les forages à travers le barrage avaient été poussés jusqu'à une profondeur dans le rocher telle que celui-ci se présente sous forme compacte et pratiquement étanche. Le travail confortatif pouvait être considéré comme terminé et donnant toute satisfaction. Depuis une surveillance régulière des piézomètres ainsi que des mesures topographiques systématiques ont permis de constater que la situation n'avait pas changé. La nature du coulis utilisé pour cette injection a eu certainement sa part dans la réussite de ce travail. C'était un coulis aéré: des micro-bulles d'air sont incluses au mélange ciment-bentonite grâce à un malaxage violent en présence d'un agent tensio-actif (alkylary sufonate). Ce chantier fut une des toutes premieres applications de ces coulis aérés dont les avantages sont une grande stabilité et une meilleure injectabilité que les coulis classiques. Bien entendu, toutes choses égales par ailleurs, les résistances en compression et traction sont minorées. Aussi, afin de ne pas trop influencer les caractéristiques mécaniques, le taux d'air incorporé a-t-il été limité à une vingtaine de pourcents.

On pourrait, bien entendu, donner beaucoup d'autres exemples de réparation de béton par injection. Les deux qui ont été exposés ci-dessus sont très caractéristiques des différentes méthodes applicables: les résines pour les fissures fines et les coulis de ciment, ou ciment-bentonite pour des vides plus importants tels que ceux résultant d'une dissolution.

Ces deux exemples sont assez anciens. Les auteurs les ont choisis intentionnellement de préférence à d'autres peutêtre plus spectaculaires pour montrer dans ces deux cas remontant à une dizaine d'années, la pérennité et la fiabilité de ces méthodes d'injection. 